\title{
INTER-PROCESS COMMUNICATION FOR FORCE DISPLAY OF DYNAMIC VIRTUAL WORLD
}

\author{
Shoichi HASEGAWA \\ Precision and Intelligence Laboratory \\ Tokyo Institute of Technology \\ 4259 Nagatsuta, Midoriku, Yokohama, 226-8503 \\ Japan \\ Email: hase@pi.titech.ac.jp
}

\author{
Masahiro ISHII \\ Yasuharu KOIKE \\ Makoto SATO \\ Precision and Intelligence Laboratory \\ Tokyo Institute of Technology \\ 4259 Nagatsuta, Midoriku, Yokohama, 226-8503 \\ Japan \\ Email: mishii, koike, msato@pi.titech.ac.jp
}

\begin{abstract}
This paper proposes a novel inter-process communication, between a virtual world manager with slow update rate and a haptic controller with fast update rate, which incorporates the integrated force given from the user to the world. High-speed refresh rate, generally $1 \mathrm{kHz}$, is required to present stiff virtual objects that have spring-damper characteristic with haptic display. On the other hand, the managing of an interactive and rich virtual world that takes a lot of computational time makes the refresh rate as low as $10 \mathrm{~Hz}$, which is enough for perceptual cycle.

The dividing of the process into two has been proposed to interact with such a world and inter-process communication between the processes has been discussed. Although the methods proposed previously work well on condition that the virtual world is static, goes wrong on dynamic world.

The importance of the force integration on the object motion acquisition is described and an inter-process communication that cares about the force integration is proposed to remove the influence of the sampling timing. An experimental result shows that the proposed method improve the accuracy of the operation.
\end{abstract}

\section{INTRODUCTION}

High-speed refresh rate generally $1 \mathrm{kHz}$ is required to present stiff virtual objects that have spring-damper character with haptic display. Lonnie and Wayne (1) reported that the update rate of $100 \mathrm{~Hz}$ for $6.7 \mathrm{kN} / \mathrm{m}$ stiffness or $1 \mathrm{kHz}$ for $70 \mathrm{kN} / \mathrm{m}$ is necessary for stable operation.

Chang and Colgate (2), using a haptic display, made a two-dimensional virtual world that consists of three fixed and four movable objects with $1 \mathrm{kHz}$ updating. Ruspini and Khatib (3) made three-dimensional dynamic models for haptic display. Their system calculates dynamics at $1 \mathrm{kHz}$ update. Nahvi et al. (4) have proposed a haptic controller, which calculates dynamic force from the motion of the virtual mechanism. Their haptic controller employs inverse dynamics and can present simple mechanisms whose degrees of freedom is less than the degrees of freedom of haptic interface. In These systems, the cycle of interaction (getting finger position, updating dynamic models, and giving haptic sensation) is managed in the same process. More complex virtual world that takes a lot of computational time must make the refresh rate slow.

\subsection{Dividing haptic controller from world manager}

The dividing of the process into two, to manage virtual world with low update rate and to control haptic display with high update rate, has been proposed to make more complex world, and inter-process communication between the world manager and the haptic controller has been discussed (5)(6)(7). With these previous works, the world manager converts the local feature of the space near the user's finger into the intermediate representation (e. g. some primitives like planes, spheres...) and sends them to the haptic controller. The haptic controller generates the force that acts on the user's finger based on the intermediate rep- 
resentation with high update rate.

Complex dynamics requires much computation time. Therefore, high performance haptic controller will be needed. If the world manager calculates dynamics at low update rate, the load of the haptic controller will be reduced. More over, the total computation time will also be reduced. Therefore, the calculation of the complex dynamics must be done by the world manager. Brooks et al. (8) reported that it is enough for the haptic interfaces for tele-operation systems to send the information of the position to the slave manipulator with update rate of 5 to $10 \mathrm{~Hz}$. It means that the high update rate like $1 \mathrm{kHz}$ is not required for the interaction between human and virtual object, but required for the stable present of stiff surface. Therefore, the calculation of the dynamics will be able to be done with low update rate by the world manager.

Ruspini and Khatib (3) proposed proxy-blending technique for complex dynamic models with slow update. However, their system treats soft objects only.

\subsection{Problem of coarse sampling}

Although previous haptic system (5)(6)(7) (3) generally works well on condition that the virtual world is static or the virtual objects are soft, goes wrong for stiff objects on dynamic virtual world.

Imagine, to understand easily, that a finger is flicking a movable virtual object floated in the virtual world. The movement of the object after the flicking depends on the timing of sampling to measure the finger position. The difference between fig.1-A and fig.1-B is sampling timing (phase). The sampling rates are same. The finger moves on equal terms. The reflection forces, which act on the user, are same in both cases. In the case of fig.1- A, the flicking is detected and the object moves. On the other hand, in the case of fig.1-B, the flicking is not detected and the object does not move. We call the instability caused by timing difference of sampling "sampling coherency problem" in this paper.

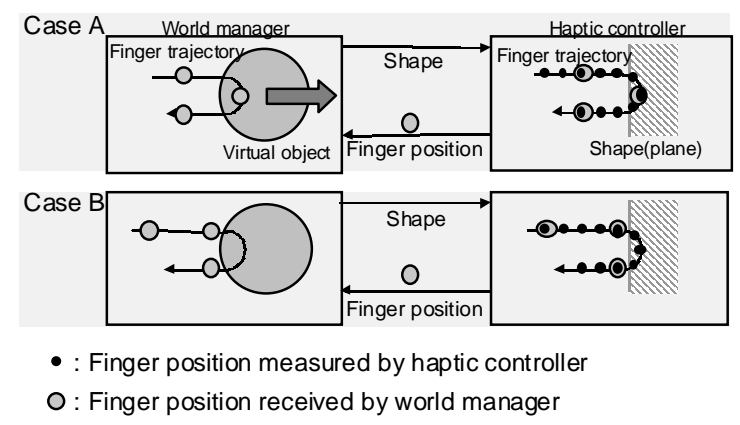

Figure 1. The influence of coarse sampling
The world manager sends rich information, or the feature of the shape. While the haptic controller renders the haptic sensation based on the feature of the shape. However, the haptic controller sends poor information, or coarse sampled finger position, back to the world manager. If the haptic controller sends more rich information back to the world manager, the intention of the user will be transmitted correctly to the virtual world.

\subsection{The purpose of this paper}

The purpose of this paper is to construct a haptic display system, which can present a virtual world consists of many dynamic objects, which can't be updated fast.

This paper explains the information, which is required for the stable update of dynamic virtual world, and proposes a novel haptic controller, which sends rich information back to the world manager.

\section{HAPTIC SYSTEM WITH FORCE INTEGRATION}

The sampling coherency problem can be solved if the haptic controller sends the finger trajectory, which is measured by haptic controller with fast update rate. However, this causes two problems.

1. The quantity of the communication will be increased.

2. The world manager must analyze the trajectory. In addition, the analysis increases computation resources consumed by world manager.

So, we think about the information, which is required for the stable update of the motion of the object.

\subsection{Object motion and force integration}

This section describes that the object motion can be decided by force integration (i.e. impulse, impetus).

Notation:

$m$ : the mass of the object.

I: the matrix of inertia $(3 \times 3$ matrix $)$.

$v$ : the velocity of the center of the gravity.

$r$ : the position of the center of the gravity.

$\omega$ : the angular velocity of the object.

$f$ : the force acts on the object.

$p$ : the position where $f$ acts on.

Dynamics of a rigid body is represented by:

$$
m \frac{d v}{d t}=f
$$

$$
I \frac{d \omega}{d t}=(p-r) \times f
$$


Integrate equation (1) from $t$ to $t+\Delta t$ :

$$
\int_{t}^{t+\Delta t} m \frac{d v}{d t} d t=\int_{t}^{t+\Delta t} f d t
$$

The velocity of the center of the gravity of the object at the next update is:

$$
v(t+\Delta t)=v(t)+\frac{1}{m} \int_{t}^{t+d t} f d t
$$

Integrate equation (2) from $t$ to $t+\Delta t$ :

$$
\int_{t}^{t+\Delta t} \frac{d I \omega}{d t} d t=\int_{t}^{t+\Delta t}(p-r) \times f d t
$$

In this case, $\Delta t$ is small and $\mathrm{p}$ and $\mathrm{r}$ are considered to be constant.

$$
\Delta I \omega=\left((p-r) \times \int_{t}^{t+\Delta t} f d t\right)
$$

The angular momentum of the object at the next update is:

$$
I(t+\Delta t) \omega(t+\Delta t)=I(t) \omega(t)+\left((p-r) \times \int_{t}^{t+d t} f d t\right)
$$

The motions of the objects depend on the force integration.

\subsection{Proposal of inter-process communication}

As described in section 2.1, instant force is not important but the integrated force is important to acquire the motion of the object. To remove the sampling coherency problem, we propose an inter-process communication, in which haptic controller sends back the information on the force integration to the world manager. Fig. 2 contrasts previous and proposed inter-process communication.

The proposed haptic controller integrates the force given from the user to the haptic display during the cycle of the world manager, and sends the information on the integrated force back to the world manager. The world manager updates the virtual world with the information.

The following is the update procedure of proposed haptic system.

1. World manager: Find the feature of the shape from the virtual world.
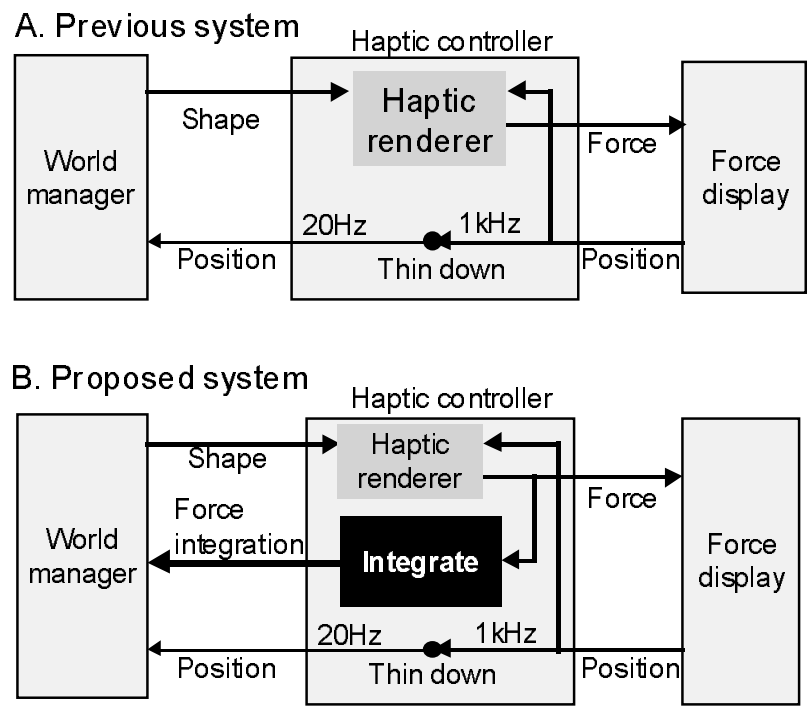

Figure 2. Haptic system with and without force integration

2. World manager: Send the feature of the shape to the haptic controller.

3. Haptic controller with high update rate:

(a) Measure finger position.

(b) Interpolate feature of the shape to present the user.

(c) Render the haptic sensation.

(d) Integrate the presented force.

4. Haptic controller: Send the information on the force integration and finger position to the world manager.

5. World manager: Update the virtual world based on the force integration.

\subsection{Motion of flicked object with proposed method}

Fig. 3 shows the effect of the proposed method on the motion of the flicked object.

In previous haptic system, the world manager calculates the penetration of the finger based on the received finger position. Then the world manager update the velocity of the object based on the penetration, which is represented by the length of $d$ in fig.3. Therefore, the velocity of the object depends on the sampling timing. In proposed haptic system, the haptic controller calculates the force integration, which is represented by the area of $s$ in fig.3. Therefore, the proposed system solves the sampling coherency problem.

\section{DETAIL OF THE PROPOSED HAPTIC SYSTEM}

We constructed a haptic system with proposed method. Following is the detail of the constructed haptic system. 
Previous method

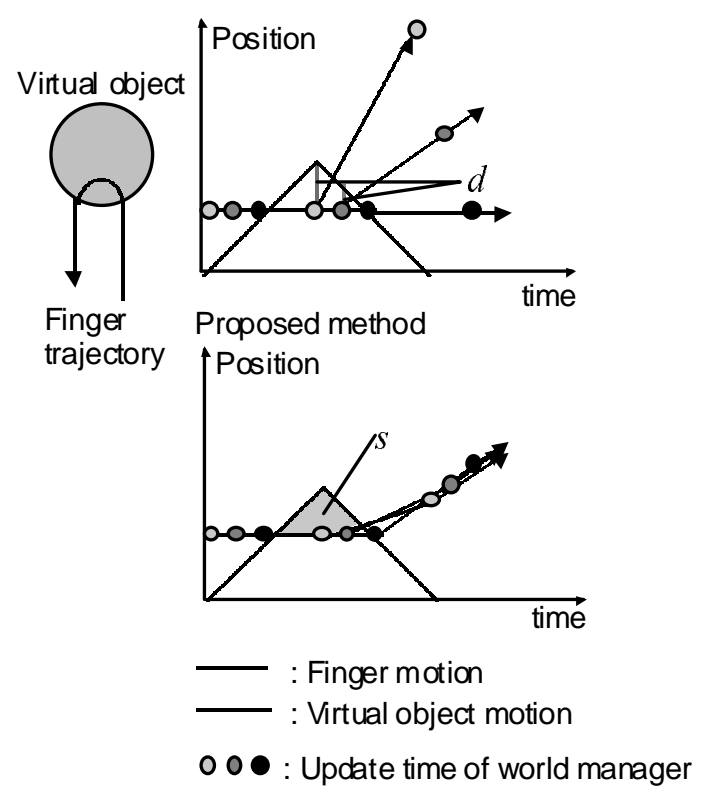

Figure 3. Motion of flicked object in the previous and proposed system

\subsection{Preparation of feature of the shape}

The world manager prepares the feature of the shape to inform to the haptic controller. In the constructed system, we employ some planes as the feature of the shape. The following is the procedure of the preparation of planes.

1. Find objects located within a certain radius sphere whose center is the finger.

2. On each object found by step 1, find the nearest point from the finger (see fig.4-2).

3. For each object found by step 1, find the plane that goes through the point found by step 2 and faces to the finger (see fig.4-3).

It is noted that the word "plane" means the pair of the point found in step 2 and the normal found in step 3 .

\subsection{Interpolation of the feature of the shape}

The update rate of the information on the feature of the shape is lower than the update rate of the haptic controller. If the haptic controller renews the feature of the shape crudely informed by the world manager, the force presenting to the user can suddenly change on a large scale and the presenting shape can be felt chattered. Therefore, the haptic controller need to interpolate received feature of the shape to show a continuous motion of the shape.

In the constructed system, we employ some planes as the feature of the shape.

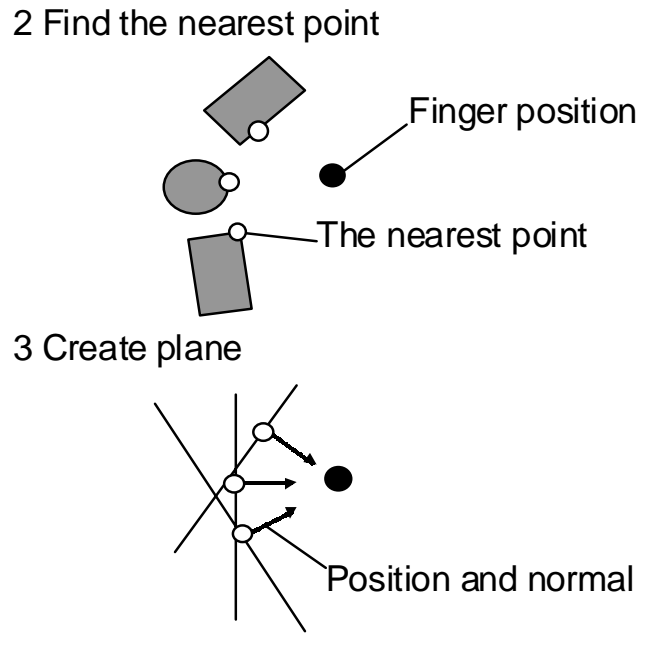

Figure 4. Preparation of planes

Notation:

$i$ : The update counter of world manager $(20 \mathrm{~Hz})$.

$j$ : The update counter of haptic controller $(1 \mathrm{kHz})$.

$P_{W_{i}}$ : Position of the plane sent from the world manager at the $i$ th update.

$N_{W_{i}}$ : Normal of the plane sent from the world manager at the $i$ th update.

$P_{H_{n i+j}}$ : Position of the plane interpolated by haptic controller at the $n i+j$ th update.

$N_{H_{n i+j}}$ : Normal of the plane interpolated by haptic controller at the $n i+j$ th update.

Here, the haptic controller updates $n$ times for one update of the world manager. While the world manager updates from $i$ th to $i+1$ th update, the haptic controller updates from $n i$ to $n i+n-1$ th update. The interpolated plane can be written in following.

$$
P_{H_{n i+j}}=\frac{j}{n} P_{W_{i}}+\frac{n-j}{n} P_{W_{i-1}}
$$

$$
N_{H_{n i+j}}=\frac{j}{n} N_{W_{i}}+\frac{n-j}{n} N_{W_{i-1}}
$$

This interpolation applies to each received plane by the haptic controller.

\subsection{Haptic rendering}

The haptic controller calculates the force presenting to the user based on the interpolated feature of the shape and measured finger position of the user.

In the constructed system, spring-damper model (9) is employed and the force generated is proportion to the penetration of 
the finger. The force presenting to the user can be represented as following.

Notation:

$\Delta t$ : The update period of the haptic controller.

$f_{j}$ : The force presented to user.

$k_{p}, k_{d}$ : Spring and damper coefficient.

$C_{j}$ : Finger position of the user.

$D_{j}$ : The nearest point to the user's finger in the area of outside of all planes.

The nearest point $D_{j}$ is calculated from the several interpolated planes (several pair of $P_{H_{j}}$ and $N_{H_{j}}$ ) based on God-object algorithm proposed by Zilles and Salisbury (10).

$$
f_{j}=k_{p}\left(D_{j}-C_{j}\right)+k_{d} \frac{\left(\left(D_{j}-C_{j}\right)-\left(D_{j-1}-C_{j-1}\right)\right)}{\Delta t}
$$

\subsection{Integration of force}

During the cycle of the world manager, the haptic controller integrates the force given from the user to the world. The amount of the force given by the user can be considered equivalent to the amount of the force generated by the haptic controller, and the direction of the forces is opposite.

The force integration is represented as following. Notation:

$F_{i}$ : Force integration, which is sent to the world manager at the $i$ th update of the world manager.

$$
F_{i}=\sum_{j=0}^{n-1} f_{n(i-1)+j}
$$

\subsection{Update of the virtual world}

The world manager updates the motion of the virtual object based on the received force integration. In the constructed system, virtual objects are treated as solid objects. Following represents the update of the motion of an object.

Notation:

$m$ : the mass of the object.

$I$ : the matrix of inertia $(3 \times 3$ matrix $)$.

$r_{i}$ : the position of the center of the gravity.

$v_{i}$ : the velocity of the center of the gravity.

$\omega_{i}$ : the angular velocity of the object.

$p_{i}$ : the position where $F_{i}$ acts on.

$$
\begin{aligned}
v_{i} & =v_{i-1}+\frac{1}{m} F_{i} \\
I_{i} \omega_{i} & =I_{i-1} \omega_{i-1}+\left(\left(p_{i}-r_{i}\right) \times F_{i}\right)
\end{aligned}
$$

\section{EVALUATION}

\subsection{Computer simulation of the motion of flicked ob- ject on the floor}

A computer simulation was done to evaluate the effect of the proposed method. The situation of the simulation was that the user's finger flicks a virtual object. In Fig.5, we applied only intermediate representation in the case of $\mathrm{A}$, and we applied not only intermediate representation but also integration of the force in B. Both graphs showed five lines that represented the motion of the virtual object. The differences of these five dotted lines were the sampling timing (phase) of the world manager. The simulation result shows that the proposed method, in case of B, had less influence of sampling timing compared to the previous method, in the case of $\mathrm{A}$.

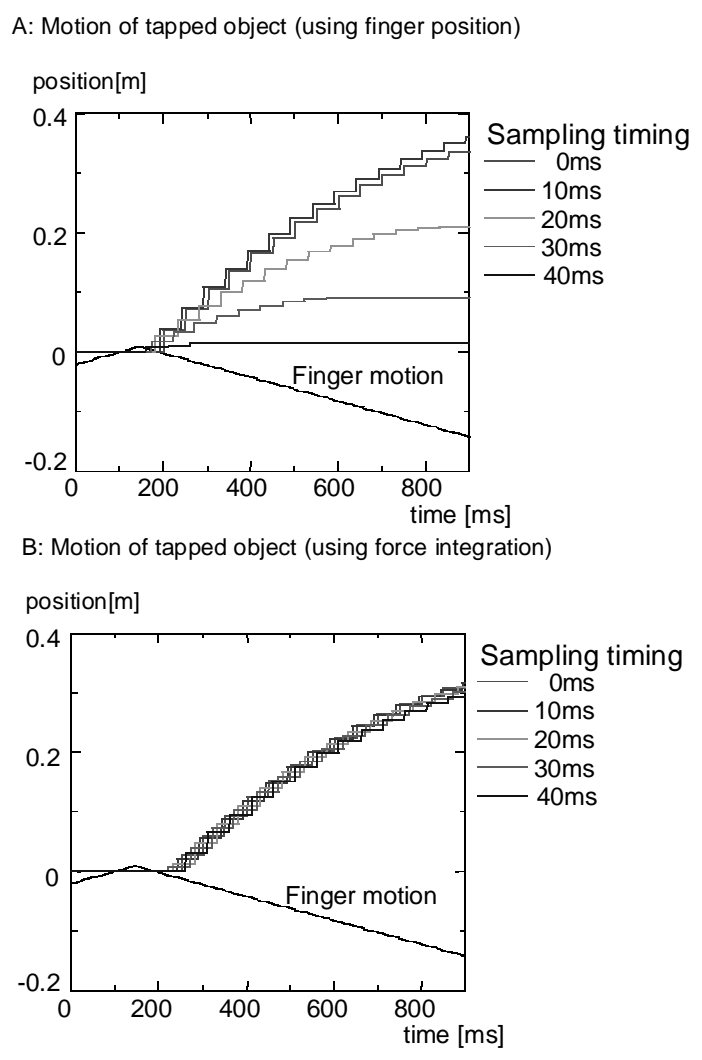

Figure 5. Simulation result

\subsection{Experiment}

4.2.1 Experimental system An experimental system had been constructed. The haptic interface device used here was SPIDAR (11) using tensed strings. A PC with Pentium-II 
$300 \mathrm{MHz}$ was used for managing world, controlling haptic interface, and rendering computer graphics.

The update frequency of the world manager was $20 \mathrm{~Hz}$ and that of the haptic controller was $1000 \mathrm{~Hz}$. The haptic controller showed 50 interpolated planes in a cycle of the world manager. The stiffness of the spring-damper model was $2 \mathrm{kN} / \mathrm{m}$. The virtual world was constructed with a three-dimensional physical simulator; the gravity, aerial resistance, and collisions and frictions among the objects were also taken in to consider.

4.2.2 Experimental task An experiment had been carried out and showed the effectiveness of the proposed method. The experimental task was as following. In the virtual world, a cube of $1 \mathrm{~kg}$ was placed on a floor at the distance scale of $0 \mathrm{~cm}$ (Fig.6). Subjects were asked to tap the cube once to move the cube forward to the distance scale of $30 \mathrm{~cm}$.

The experiment was done with two different (proposed and previous) methods of force calculation. Subjects completed the task with one certain method 30 times and with another method 30 times.

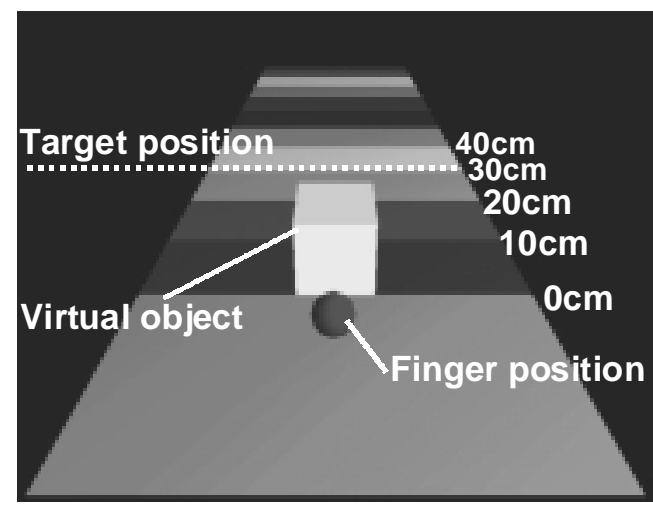

Figure 6. The picture of the experimental task.

4.2.3 Experimental result Fig.7 showed the position where the cube stopped. It showed that with the proposed method, the subject could learn how to move the cube to the correct position, but with the previous method, he could not. Fig. 8 showed the average of the deviation error of the stop position of last 20 trials. The result showed that the proposed method obviously improved the accuracy of the operation.

\section{RESULTS}

The proposed method will be able to apply to various models of dynamics. At present, we made a virtual environment with
With previous method
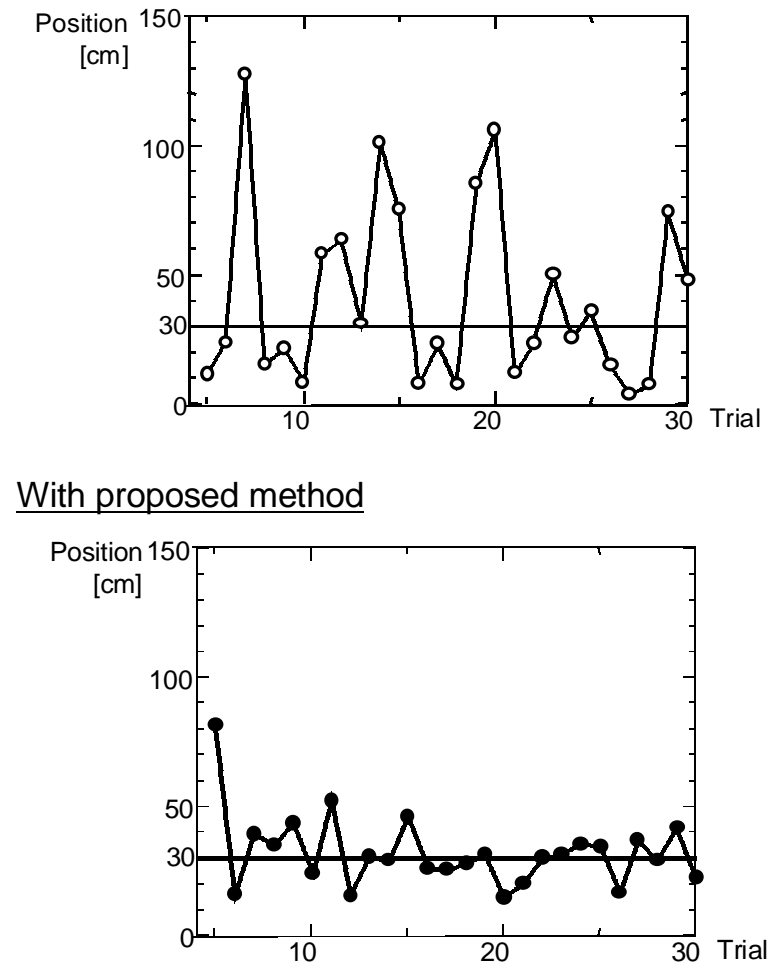

Figure 7. The stop position of the virtual object

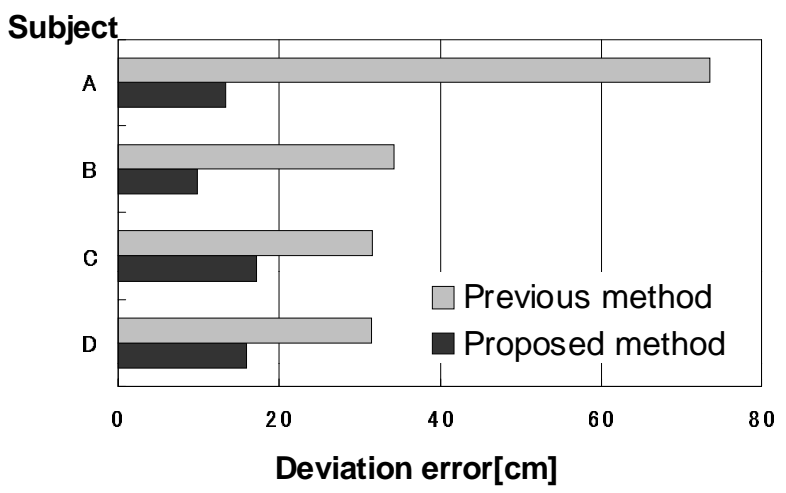

Figure 8. Compare of the accuracy of the operation

impulse-based simulator (12). More than 20 movable spheres could be put on a fixed rectangular floor in the virtual world. Each sphere had six DOF and frictions and gravity were considered. The user could both operate and feel the objects in the natural way. 


\section{DISCUSSION}

\subsection{Contrast force display and graphics display}

In graphics systems, the graphics renderer was divided from the world manager earlier than the haptic controller and special hardware were developed. Therefore, we construct division of haptic controller and that of graphics renderer.

6.1.1 Division of graphics system A graphics display is an output device to present information to the user, but not an input device. Therefore, the information flows from the virtual world to the user as shown in fig.9.

The division of the graphics system makes the information from the virtual world rich information.

6.1.2 Division of haptic system A haptic display not only displays shapes and forces but also inputs the finger position of the user and the force given by user. Therefore the flow of the information becomes both directional as shown in fig.10.

Previous haptic systems for complex virtual world employ rich information in the communication from the virtual world to the user, but employ poor information in the communication from the user to the virtual world. Therefore, previous system can not transmit user's intention correctly to the virtual world. In the proposed system, the virtual world receives rich information, or force integration. Therefore, the system can transmit the user's intention correctly.

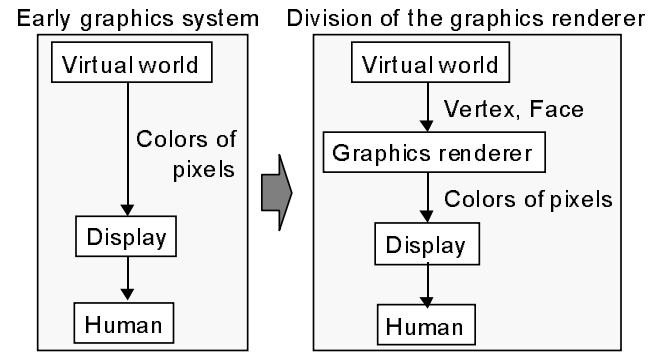

Figure 9. Process division in graphics systems

\subsection{Representation of friction}

The haptic controller interpolates the position of the plane. Because the position of the plane is the position of the point where the collision between the finger and the object may occur, the velocity of the plane is different from the velocity of the object, which is expressed by the plane. To represent friction, the world manager must send also the velocity of the object to the haptic controller. Because the relative velocity from the object to the finger becomes necessary to calculate the frictional force.

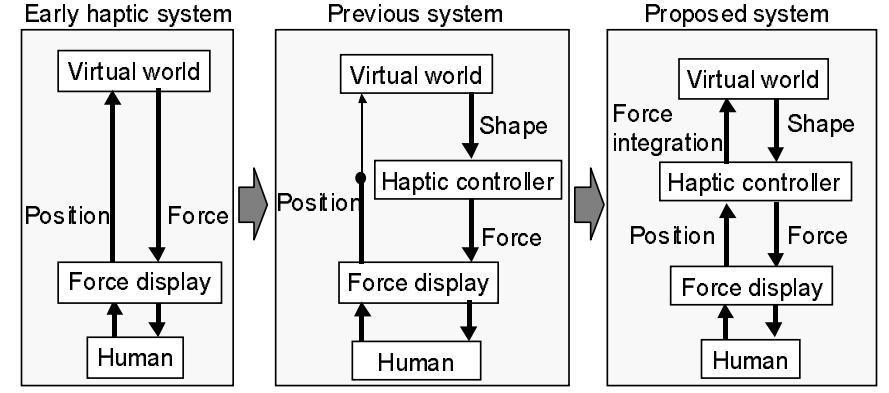

Figure 10. Process division in haptic systems

We had already loaded this idea and confirmed that the friction could be expressed.

\subsection{Layers in the haptic system}

This paper described that natural update of the virtual world can be achieved by adding a few function to the haptic controller and using rich information for the communication. Adding more function to the haptic controller and using more rich information for the communication, more natural update of virtual world will be able to achieve. Therefore, haptic system with various dividing point can be constructed (fig.11). We construct a haptic system with more functional haptic controller, which calculate some part of the motion of solid object. The haptic system improves the haptic sensation in case the world manager updates more slowly.

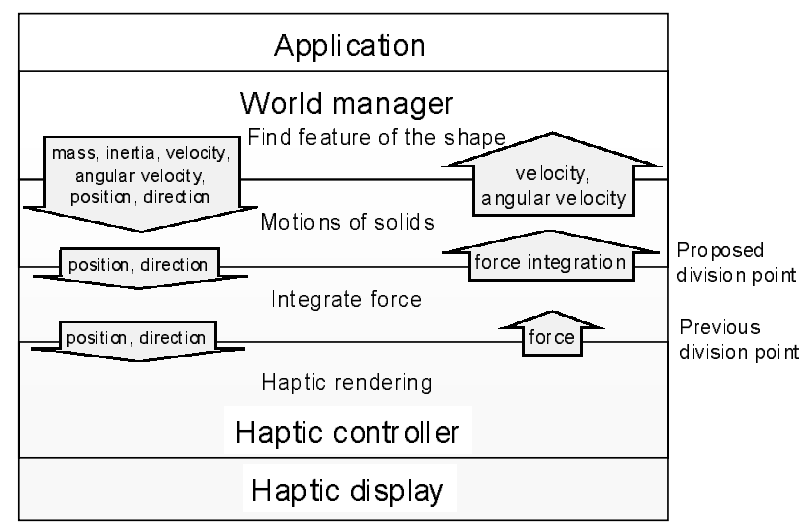

Figure 11. The function of the haptic controller and information of the communication 


\subsection{Deformation of objects}

There are some methods to deform a virtual object using haptic interface. These methods deform an object based on the penetration of the finger to the object (13). The proposed method could be able to realize subtle deformations of objects by the deformation based on the integrated force. Experiment is to be carried out to support the idea.

\section{CONCLUSION}

A new scheme of haptic V.R. system was proposed. In this scheme, the haptic controller integrates the force from user to haptic display and sends the integration of force to the world manager. It was shown that proposed method removes the sampling coherency problem. An experiment was made to check the effectiveness of the proposed method. Results showed that proposed method improve the accuracy of the operation.

\section{REFERENCES}

Lonnie Love and Wayne Book, "Contact Stability Analysis of Virtual Walls", Proc. of Dynamic Systems and Control Division ASME 1995, pp.689-694

Beeling Chang and J. Edward Colgate, "Real- time impulsebased simulation for haptic display", Proc. of the 1997 ASME International Mechanical Engineering Congress and Exhibition Diego Ruspini, Oussama Khatib, "Dynamic Models for Haptic Rendering Systems", Advances in Robot Kinematics: ARK'98, June 1998, Strobl/Salzburg, Austria, pp.523-532

Ali Nahvi, Donald D. Nelson, John M. Hollerbach, and David E. Jhonson, "Haptic Manipulation of Virtual Mechanisms from Mechanical CAD Design", Proc. of the 1998 IEEE International Conference on Robotics and Automation, pp.375-380

Adachi Y., Kumano T., and Ogino K., "Intermediate Representation for Stiff Virtual Objects”, Proc. IEEE Virtual Reality Annual International Symposium '95, pp.203-210

William R. Mark, Scott C. Randolph, Mark Finch, James M. Van Verth, and Russell M. Taylor II, "Adding Force Feedback to Graphics Systems: Issue and Solutions", SIGGRAPH 96 Conference Proc. 1996 ACM

Juli Yamashita, Cai Yi, and Yukio Fukui, "Feature-Based Haptic Rendering and Protocol", Proc. of the Virtual Reality Society of Japan Second Annual Conference, 1997, pp.195-197

Thurston L. Brooks, "Telerobotic response requirements", Proc. of the 1990 IEEE International Conference on System, Man and Cybernetics, pp.113-120

Hogan,N., "Stable execution of contact tasks using impedance control", Proc. of IEEE Robotics and Automation Conference, 1987, pp.1047-1054

C. B. Zilles, J. K. Salisbury, "A Constraint-based God-object Method For Haptic Display", ASME Haptic Interfaces for Vir- tual Environment and Teleoperator System 1994, Dynamic Systems and Control 1994, vol. 1, pp.146-150

Masahiro Ishii and Makoto Sato, "A 3D Spatial Interface Device Using Tensed Strings", Presence Vol. 3, No. 1, Winter 1994, pp.81-86

Brian Vincent Mirtich, "Impulse-based Dynamic Simulation of Rigid Body Systems", Ph.D. thesis, University of California, Berkeley, December, 1996.

Kenji Yamamoto, Akio Ishiguro, and Yoshiki Uchikawa, "A Development of Dynamic Deforming Algorithm for 3D Shape Modeling with Generation of Interactive Force Sensation", Proc. 1993 IEEE Virtual Reality Annual International Symposium, pp.505-511 